\title{
CONCENTRAÇÕES DE FIBRINOGÊNIO PLASMÁTICO, FOSFATASE ALCALINA SÉRICA E DO FIBRINOGÊNIO E FOSFATASE ALCALINA NO FLUIDO PERITONEAL DE EQUINOS SUBMETIDOS À ENTERORRAFIAS APOSICIONAL E INVAGINANTE NO CÓLON DESCENDENTE
}

\author{
VALENTE, Paula Pimentel ${ }^{1}$ \\ CATTELAN, José Wanderley ${ }^{2}$ \\ SANTANA, Aureo Evangelista ${ }^{2}$ \\ MALHEIROS, Euclides Braga ${ }^{3}$ \\ DUARTE, Claudia Acosta ${ }^{4}$ \\ RASERA, Luciane ${ }^{5}$ \\ AITA, Andréa da Costa ${ }^{6}$
}

Recebido em: 2009-10-11

Aprovado em: 2009-10-30

ISSUE DOI: $10.3738 / 1982.2278 .321$

\begin{abstract}
RESUMO: Neste ensaio foram monitoradas as concentrações do fibrinogênio plasmático e da fosfatase alcalina sérica e do fibrinogênio e fosfatase alcalina no fluido peritoneal (FP), nos períodos pré e pós operatórios, de equinos submetidos a enterorrafias invaginante (Grupo I) e aposicional (Grupo II) no cólon descendente. Foram utilizados 14 eqüinos adultos, sem raça definida, hígidos e com idade entre cinco e oito anos, submetidos à laparotomia de flanco esquerdo. Foram colhidas amostras de sangue e de fluido peritoneal nos períodos pré-jejum (-24 horas), pré-cirúrgico (0h) e às 6, 12, 18, 24, 48, 72, 96, 168, 336, 504, 672, 840, 1008 e 1176 horas de pós-operatório (p.o.) e realizadas as mensurações da concentração de fibrinogênio e da atividade da fosfatase alcalina. Nos dois grupos de equinos submetidos a enterotomias do cólon descendente foi possível evidenciar que as alterações nos parâmetros de fibrinogênio e atividade da fosfatase alcalina são mensuradas mais precocemente no fluido peritoneal do que no sangue circulante.
\end{abstract}

Palavras- chave: Eqüinos. Fibrinogênio. Fosfatase alcalina. Cólon descendente

SUMMARY: This study monitorated during the pre and postoperative periods the concentrations of plasma fibrinogen, serum alkaline phosphatase, fibrinogen and alkaline phosphatase in peritoneal fluid of horses submitted to enterorrafies of descending colon closed by full-thickness and simple continuous pattern (Group I) and directly apposition of the bowel wall using a single layer (Group II). Fourteen healthy, adult, undefined breed horses, between five and eight year-old were submitted a left flank laparotomy. Samples of blood and peritoneal fluid were collected at prefasting ( 24 hours), preoperative (0 hour) and at $6,12,18,24,48,72,96,168,336,504,672,840,1008$ and 1176 hours of postoperative periods for determination of fibrinogen concentration and the activity of alkaline phosphatase. Results of both groups from equines submitted to enterorrafies of descending colon showed that changes in fibrinogen and alkaline phosphatase parameters should be identified earlier in peritoneal fluid (6 hours p.o) than in blood samples.

Keywords: Equine. Fibrinogen. Alkaline phosphatase. Descending colon

\footnotetext{
${ }^{1}$ Profa. Faculdade Dr Francisco Maeda e Centro Universitário Moura Lacerda- Mestre em Cirurgia Veterinária- SP. ${ }^{2}$ Professores Doutores do Departamento de Clínica e Cirurgia Veterinária - FCAV - UNESP - Jaboticabal - SP

${ }^{3}$ Professor Doutor do Departamento de Estatística - FCAV - UNESP - Jaboticabal - SP.

${ }^{4}$ Professora Doutora do Departamento de Cirurgia Veterinária da Universidade Federal do Pampa- Uruguaiana - RS

${ }^{5}$ Professora Doutora do Departamento de Cirurgia Veterinária - FAJ- Jaguariúna- SP.

${ }^{6}$ Médica Veterinária Autônoma- Mestre em Cirurgia Veterinária - Brasília-DF.
} 


\section{INTRODUÇÃO}

As afecções gastrintestinais em equinos são atribuídas a causas diversas e complexas (WHITE II, 1990) podendo apresentar sinais clínicos similares, porém os prognósticos podem ser distintos (DAVIES et al., 1984). A prevalência das afecções do cólon descendente perfaz um total de 3 a $18 \%$ dos casos de lesões gatrintestinais específicas (WHITE II, 1990) e são vários os fatores que predispõem a altas taxas de complicações pós-cirúrgicas em afecções deste segmento intestinal (STASHAK, 1982; KELLER; HORNER, 1985 citados por EDWARDS, 1997).

O fluido peritoneal reflete as condições e as alterações que ocorrem na superfície mesotelial da cavidade abdominal (BACCARIN et al., 1995) e o estudo de suas características físico-químicas e citológicas torna-se importante e indispensável na avaliação de equinos com lesões abdominais (MALARK et al., 1992; VALADÃO et al.1996).

Alterações encontradas no fluido peritoneal podem refletir inflamações encontradas em decorrência de procedimento cirúrgico abdominal ou devido a peritonite, (FISHER JR. et al., 1986; BLACKFORD et al., 1986; SANTSCHI, et al.,1988; SCHNEIDER et al., 1988), porém não há relatos de quanto tempo a inflamação pós-operatória persiste.

Por meio da abdominocentese é possível monitorar pacientes que apresentam cólica intermitente ou crônica, diarréia aguda ou crônica, perda de peso persistente, ascite, suspeita de ruptura de bexiga em potros (BARRELET, 1993) e pacientes que desenvolvem peritonite natural ou secundária a intervenções cirúrgicas abdominais (BLACKFORD et al., 1986), ou ainda, em decorrência de ruptura intestinal (HAWKINS et al., 1993).

Tem sido observada correlação entre os constituintes do plasma e fluido peritoneal, devido à livre difusão entre os compartimentos, visto que o fluido peritoneal trata-se de um dializado plasmático (BROWNLOW et al., 1981; PARRY; BROWNLOW, 1992). Essa correlação é facilmente verificada nas taxas de uréia, glicose, bilirrubina e fósforo inorgânico, entretanto, no que concerne aos níveis de proteínas totais, albumina, cálcio, aspartato amino transferase e fosfatase alcalina tal correlação não é comumente observada (BROWNLOW et al., 1981). Valores elevados de fosfatase alcalina no fluido peritoneal na ausência de aumento correspondente no soro de equinos portadores de lesões abdominais foram relatados por FROSCHER e NAGODE (1979). 
A literatura consultada revela valores dos constituintes do líquido peritoneal tanto em animais sadios quanto em portadores de distúrbios abdominais, que são utilizados rotineiramente como parâmetros de referência com intuito diagnóstico. Porém, são escassos os valores de referência obtidos a partir de animais submetidos a intervenções cirúrgicas, inclusive enterotomias e durante um período longo no pós-operatório. Faleiros ( 2003 ) ao realizar enterotomias no cólon descendente de equinos avaliou as concentrações de proteína total, fibrinogênio, lactato e glicose no sangue e no fluido peritoneal durante 3 horas pós-operatórias, entretanto os valores de fosfatase alcalina não foram avaliados. Portanto, este ensaio teve o propósito de comparar as alterações das concentrações sanguíneas e do fluido peritoneal de fibrinogênio e da fosfatase alcalina em equinos submetidos a enterorrafias aposicional e invaginante no cólon descendente.

\section{MATERIAL E MÉTODOS}

Foram utilizados 14 eqüinos adultos hígidos, sete machos e sete fêmeas, sem raça definida e com idade entre cinco e oito anos, submetidos a jejum alimentar de 24 e hídrico de 12 horas previamente à intervenção cirúrgica. A tranqüilização dos pacientes foi realizada com cloridrato de xilazina ${ }^{7}(1,1 \mathrm{mg} / \mathrm{Kg})$, o derrubamento com éter gliceril guaiacol $^{8}(110 \mathrm{mg} / \mathrm{Kg})$ e a indução anestésica com tiopental sódico ${ }^{9}(12,5 \mathrm{mg} / \mathrm{Kg})$, todos por via intravenosa. A anestesia geral inalatória foi feita com halotano ${ }^{10}$ vaporizado em oxigênio, em circuito semi-fechado.

$\mathrm{O}$ acesso à cavidade abdominal foi realizado de acordo com o procedimento cirúrgico descrito por Turner e McIlwraith (1982). O cólon descendente foi exteriorizado e isolado com compressas estéreis e o conteúdo intestinal foi comprimido e deslocado manualmente em sentido caudal. Foi feita enterotomia de $5 \mathrm{~cm}$ de extensão, localizada a $40 \mathrm{~cm}$ caudal ao ligamento duodenocólico, de acordo com a técnica descrita por Archer et al. (1988). A incisão foi executada longitudinalmente sobre a taenia coli, conforme indicação de STASHAK (1982) e a ferida intestinal suturada com fio

\footnotetext{
${ }^{7}$ ROMPUM- BAYER;

${ }^{8}$ ÉTER GLICERIL GUAIACOL- HENRYFARMA LTDA;

${ }^{9}$ TIOPENTAL- CRISTÁLIA;

${ }^{10}$ HALOTANO- CRISTÁLIA;
} 
absorvível sintético de poliglactina $910 \mathrm{n}^{\circ} 0^{11}$, segundo Lucas (2001). No primeiro grupo experimental (G I), composto por sete equinos, a enterorrafia empregada foi a contínua simples perfurante total com aposição das bordas no primeiro plano e sutura invaginante padrão Cushing no segundo plano, enquanto que no segundo grupo (G II), com número idêntico de pacientes, a enterorrafia foi executada em padrão aposicional perfurante total com pontos interrompidos simples, usando o mesmo material de síntese. Finalizada a sutura, irrigou-se o local da enterotomia com solução estéril de cloreto de sódio a $0,9 \%$ a $37^{\circ} \mathrm{C}$, e em seguida, o cólon descendente foi reintroduzido na cavidade abdominal. A laparorrafia foi realizada conforme a técnica indicada por Turner e McIlwraith (1982), empregando-se o fio de náilon $\mathrm{n}^{0} 1^{12}$ com sutura festonada nos planos musculares e contínua simples na pele.

A terapia pós-cirúrgica imediata consistiu na administração de penicilina procaína $^{13}$, conforme McIlwraith e Turner, (1987) e sulfato de gentamicina ${ }^{14}$ conforme $^{2}$ Godber et al., (1995) durante cinco dias consecutivos. Os curativos da ferida cirúrgica foram realizados diariamente com polivinilpirrolidona-iodo tópico ${ }^{15}$ a $1 \%$ até a retirada dos pontos cutâneos.

O sangue venoso foi obtido por punção da jugular e as amostras de soro foram colhidas em tubos estéreis para realização da análise bioquímica de determinação da fosfatase alcalina.

As amostras de plasma para mensuração da concentração de fibrinogênio foram colhidas em frascos contendo citrato com a utilização de kit específico ${ }^{16}$ e a leitura dos resultados com coagulômetro $(1765)^{17}$.

As colheitas de líquido peritoneal foram realizadas conforme a técnica descrita por White II (1990) com cânulas mamárias estéreis e colhidas em frascos estéreis para a realização do exame da fosfatase alcalina com a utilização de kits específicos e leitura espectrofotométrica ${ }^{18}$.

As amostras de fluido peritoneal (FP) para mensuração da concentração de fibrinogênio foram colhidas em frascos contendo citrato com a utilização de kit

\footnotetext{
${ }^{11}$ VYCRIL- ETICON;

${ }^{12}$ MONONYLON- ETHICON

${ }^{13}$ AGROVET- CIBA

${ }^{14}$ GENTOCIN- SCHERING- PLOUGH

${ }^{15}$ POVIDINE TÓPICO- JOHNSON

${ }^{16}$ ORGANON- ALEMANHA

${ }^{17}$ COAGULATOR- ORGANON- TEKNIKA- ALEMANHA

${ }^{18}$ LABQUEST- LABTEST DIAGNÓSTICA- MG.
} 
específico $^{19}$ e a leitura dos resultados com coagulômetro $(1765)^{20}$.

As amostras foram processadas imediatamente após as colheitas.

As colheitas de sangue e de líquido peritoneal foram realizadas nos períodos préjejum (-24h), pré-cirúrgico (0h) e às 6, 12, 18, 24, 48, 72, 96, 168, 336, 504, 672, 840, 1008 e 1176 horas de pós-operatório (p.o.).

Os valores obtidos foram submetidos à análise de variância em delineamento inteiramente casualizado (DIC), usando esquema de parcela subdividida no tempo, empregando-se o programa SAS (Statistical Analysis Systems), seguida da comparação das médias pelo teste Tukey $(\mathrm{P} \leq 0,05)$ para os diferentes momentos.

\section{RESULTADOS E DISCUSSÃO}

Após a análise laboratorial do sangue periférico observaram-se diferenças $(\mathrm{p} \leq 0,05)$ nos valores médios das variáveis avaliadas, em função do tempo, em todos os animais.

Os valores médios do fibrinogênio plasmático apresentaram-se aumentados a partir das 48 horas pós-operatórias, atingindo valores máximos às 96 e 168 horas e, a partir das 336 horas p.o., retornaram aos valores iniciais (Tabela 1). Nos demais momentos não foram evidenciadas diferenças $(\mathrm{P} \leq 0,05)$ entre os grupos I e II.

Neste estudo, todas as amostras foram colhidas com sucesso, diferindo de autores que relataram dificuldades na obtenção de amostras do fluido peritoneal de equinos no período pós-operatório (BLACKFORD et al. 1986), em casos de cólica (TULLENERS, 1983) e em animais sadios (BROWNLOW et al. 1981).

Os valores médios de fibrinogênio do fluido peritoneal (FP) aumentaram $(\mathrm{P} \leq 0,05)$ nas primeiras 6 horas p.o. e mantiveram-se crescentes até adquirirem valores máximos às 18,72 e 96 horas, retornando aos iniciais às 168 horas p.o. (Tabela 1).

A mensuração da concentração do fibrinogênio plasmático torna-se importante tanto para avaliação da coagulação sanguínea como em processos inflamatórios (KANEKO; SMITH, 1967 citados por KANEKO, 1989), visto que nestas situações estes valores encontram-se aumentados (COFFMAN, 1979; PARRY; BROWNLOW, 1992). O aumento nos valores médios totais de fibrinogênio plasmático às $18,48,72 \mathrm{e}$

\footnotetext{
${ }^{19}$ ORGANON- ALEMANHA

${ }^{20}$ COAGULATOR- ORGANON- TEKNIKA- ALEMANHA
} 
96 horas p.o. confirmou a presença de processo inflamatório até a $96^{\mathrm{a}}$ hora p.o., ratificando as observações dos autores supracitados.

Os valores médios de fibrinogênio do fluido peritoneal apresentaram aumentos expressivos nas primeiras 6 horas p.o., atingindo o pico às 18, 48, 72 e 96 horas, com retorno aos valores iniciais às 168 horas p.o., corroborando Santschi et al. (1988), que também observaram aumento nos valores de fibrinogênio a partir das 6 horas p.o. até às 144 horas p.o., pois esta foi a última colheita realizada pelos autores. Já Faleiros (2003) ao realizar enterotomias e induzir distensão intraluminal no cólon menor não evidenciou alterações no fibrinogênio plasmático nem do fluido peritoneal, visto que as colheitas foram realizadas até a terceira hora pós-cirúrgica. $\mathrm{O}$ aumento observado nesta pesquisa, nos valores do fibrinogênio do FP, confirma a existência de inflamação intraabdominal em decorrência do procedimento cirúrgico realizado.

Tabela 1 - Valores referentes a média geral e desvio padrão para fibrinogênio plasmático e do fluido peritoneal $(\mathrm{mg} / \mathrm{dL})$ de equinos submetidos a enterorrafias invaginante (GI) e aposicional (GII) no cólon descendente, nos diferentes momentos de colheita.

(Continua)

\begin{tabular}{|c|c|c|c|c|c|c|}
\hline & \multicolumn{3}{|c|}{ FIBRINOGÊNIO PLASMÁTICO (mg/dL) } & \multicolumn{3}{|c|}{$\begin{array}{c}\text { FIBRINOGÊNIO DO FLUIDO } \\
\text { PERITONEAL }(\mathrm{mg} / \mathrm{dL})\end{array}$} \\
\hline $\begin{array}{l}\text { TEMPO } \\
\text { (horas) }\end{array}$ & $\begin{array}{c}\text { MGG } \\
\text { GRUPO I }\end{array}$ & $\begin{array}{c}\text { MGG } \\
\text { GRUPO II }\end{array}$ & MGT & $\begin{array}{c}\text { MGG } \\
\text { GRUPO I }\end{array}$ & $\begin{array}{c}\text { MGG } \\
\text { GRUPO II }\end{array}$ & MGT \\
\hline-24 & $\begin{array}{r}249,71 \\
\pm \quad 100,56\end{array}$ & $\begin{array}{r}169,33 \\
\pm 36,74\end{array}$ & $\begin{array}{l}212,61 \\
\pm 85,72\end{array}$ & $\begin{array}{l}15,95 \\
\pm 7,74\end{array}$ & $\begin{array}{c}5,08 \\
\pm 6,00\end{array}$ & $\begin{array}{l}10,93 \\
\pm 8,76\end{array}$ \\
\hline 0 & $\begin{array}{r}208,43 \\
\pm 93,59 \\
\end{array}$ & $\begin{array}{r}191,83 \\
\pm 46,68 \\
\end{array}$ & $\begin{array}{r}200,77 \\
\pm 73,22 \\
\end{array}$ & $\begin{array}{r}12,28 \\
\pm 7,54 \\
\end{array}$ & $\begin{array}{r}3,50 \\
\pm 2,50 \\
\end{array}$ & $\begin{array}{c}8,23 \\
\pm 7,20 \\
\end{array}$ \\
\hline 6 & $\begin{array}{l}233,85 \\
\pm 123,96 \\
\end{array}$ & $\begin{array}{r}248,33 \\
\pm 111,45 \\
\end{array}$ & $\begin{array}{r}\text { abcde } \\
240,24 \\
\pm 113,64 \\
\end{array}$ & $\begin{array}{r}38,26 \\
\pm 47,14 \\
\end{array}$ & $\begin{array}{r}20,13 \\
\pm 28,07 \\
\end{array}$ & $\begin{array}{r}29,89 \\
\pm 39,09 \\
\end{array}$ \\
\hline 12 & $\begin{array}{r}238,28 \\
\pm 80,63 \\
\end{array}$ & $\begin{array}{r}240,50 \\
\pm 81,98 \\
\end{array}$ & $\begin{array}{l}\text { abcde } \\
239,31 \\
\pm 77,80\end{array}$ & $\begin{array}{r}21,43 \\
\pm 10,68 \\
\end{array}$ & $\begin{array}{r}44,00 \\
\pm 66,81 \\
\end{array}$ & $\begin{array}{r}31,84 \\
\pm 45,32 \\
\end{array}$ \\
\hline 18 & $\begin{array}{l}278,14 \\
\pm 66,54 \\
\end{array}$ & $\begin{array}{c}213,00 \\
\pm 113,52 \\
\end{array}$ & $\begin{array}{l}\text { abcde } \\
248,07 \\
\pm 93,41 \\
\end{array}$ & $\begin{array}{r}35,36 \\
\pm 17,65 \\
\end{array}$ & $\begin{array}{r}66,83 \\
\pm 77,05 \\
\end{array}$ & $\begin{array}{r}49,88 \\
+53,81 \\
\end{array}$ \\
\hline 24 & $\begin{array}{r}275,00 \\
\pm 36,85 \\
\end{array}$ & $\begin{array}{r}289,16 \\
\pm 56,60 \\
\end{array}$ & $\begin{array}{l}281,54 \\
\pm 45,58 \\
\end{array}$ & $\begin{array}{r}34,57 \\
\pm 20,90 \\
\end{array}$ & $\begin{array}{r}43,06 \\
\pm 38,53 \\
\end{array}$ & $\begin{array}{c}39,41 \\
\pm 29,44 \\
\end{array}$ \\
\hline 48 & $\begin{array}{l}307,00 \\
\pm 88,11 \\
\end{array}$ & $\begin{array}{r}292,50 \\
\pm 80,23 \\
\end{array}$ & $\begin{array}{l}300,31 \\
\pm 81,36 \\
\end{array}$ & $\begin{array}{c}54,14 \\
\pm 20,70\end{array}$ & $\begin{array}{r}33,36 \\
\pm 34,07\end{array}$ & $\begin{array}{r}44,55 \\
\pm 28,53\end{array}$ \\
\hline 72 & $\begin{array}{l}318,57 \\
\pm 90,81 \\
\end{array}$ & $\begin{array}{r}306,66 \\
\pm 101,07 \\
\end{array}$ & $\begin{array}{l}313,07 \\
\pm 91,75 \\
\end{array}$ & $\begin{array}{r}51,00 \\
\pm 18,62 \\
\end{array}$ & $\begin{array}{r}49,96 \\
\pm 32,14 \\
\end{array}$ & $\begin{array}{r}50,52 \\
\pm 24,58 \\
\end{array}$ \\
\hline 96 & $\begin{array}{l}342,14 \\
\pm 64,73\end{array}$ & $\begin{array}{l}300,83 \\
\pm 63,90\end{array}$ & $\begin{array}{l}323,07 \\
\pm 65,24\end{array}$ & $\begin{array}{c}52,00 \\
\pm 18,62\end{array}$ & $\begin{array}{l}45,56 \\
\pm 20,50\end{array}$ & $\begin{array}{c}49,03 \\
\pm 18,96\end{array}$ \\
\hline
\end{tabular}


Tabela 1 - Valores referentes a média geral e desvio padrão para fibrinogênio plasmático e do fluido peritoneal $(\mathrm{mg} / \mathrm{dL})$ de equinos submetidos a enterorrafias invaginante (GI) e aposicional (GII) no cólon descendente, nos diferentes momentos de colheita.

(Conclusão)

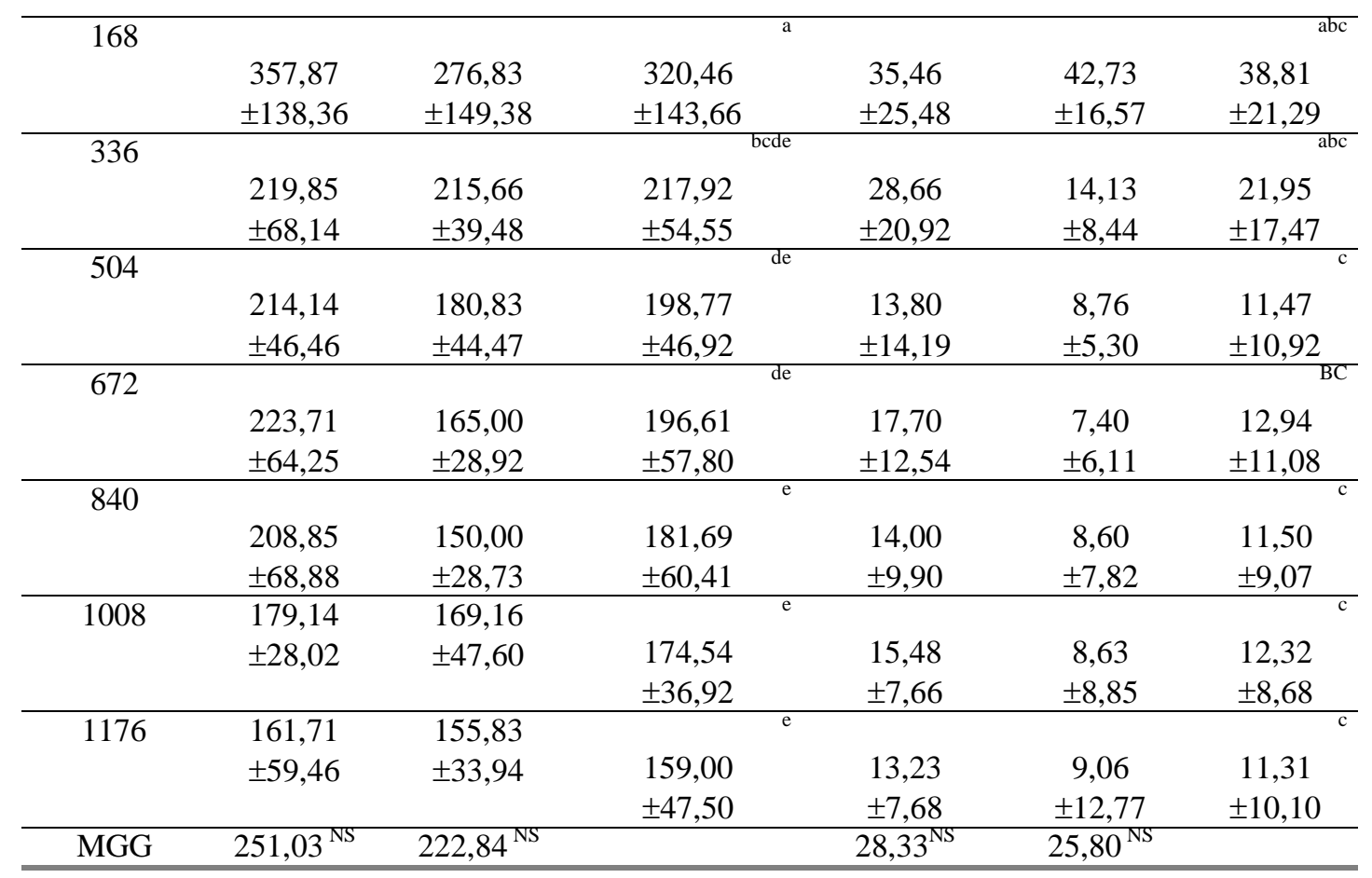

Médias seguidas pela mesma letra não diferem entre si (Teste Tukey, $\mathrm{p} \square 0,05$ )

Letras minúsculas diferentes representam diferença $(\mathrm{p} \leq 0,05)$ entre momentos

MGT= média geral dos grupos em cada momento

MGG= média geral de cada grupo em todos os momentos

Observou-se diferença entre os grupos, nos momentos pré-jejum (24 horas précirúrgicas) e pré-cirúrgico (0 hora) nos valores médios de atividade da fosfatase alcalina sérica, sendo estes maiores no grupo I. Ainda, no momento pré-cirúrgico ( 0 hora) observou-se diferença entre os grupos nos valores médios das taxas de fibrinogênio no fluido peritoneal.

Embora tenham sido encontradas diferenças entre os grupos, nos períodos préjejum e pré-operatório, tal fato, aparentemente, não apresenta significado clínico, visto que os valores mensurados são considerados normais para equinos. Nos outros momentos não foram observadas diferenças entre os Grupos I e II.

Os valores médios da atividade sérica da fosfatase alcalina apresentaram aumento discreto nas 6 primeiras horas p.o., não diferindo dos iniciais $(\mathrm{P} \square 0,05)$ durante $\mathrm{o}$ período experimental (Tabela 2).

Foi observado aumento nos valores médios da atividade da fosfatase alcalina do fluido peritoneal a partir das 6 horas p.o., atingindo valores máximos às 18 horas p.o.e, 
retorno aos valores iniciais, às 48 horas p.o. Tem sido sugerido que o aumento da atividade da fosfatase alcalina no FP indica desvitalização do intestino delgado de equinos com lesões abdominais (FROSHER; NAGODE, 1979, PEIRÓ, 1997), entretanto, a fosfatase alcalina também pode originar-se dos cólons maior e descendente, rim, fígado e leucócitos granulócitos (JAIN, 1986), dificultando a constatação da origem deste aumento. Froscher e Nagode (1979), analisaram por eletroforese, amostras de FP de equinos portadores de distúrbios abdominais e com valores aumentados para a fosfatase alcalina e concluíram que a enzima era proveniente de granulócitos em degeneração. Os valores máximos da atividade de fosfatase alcalina encontrados neste estudo chegaram a ser 10 vezes maiores que os normais da espécie, denotando a presença de injúria abdominal. Isso corrobora os achados de Silva (2005) que observou aumento seis vezes maior no grupo de equinos com cólica. Portanto, sugere-se que o aumento da atividade da fosfatase alcalina observado nesta pesquisa, possa ser atribuído à destruição de leucócitos granulócitos peritoneais após o procedimento cirúrgico realizado no cólon descendente, coincidindo com as observações de Froscher e Nagode (1979).

Por se tratar de um dialisado plasmático, os constituintes do plasma com baixo peso molecular difundem-se para a cavidade peritoneal resultando em proporções semelhantes entre dois compartimentos (BROWNLOW et al., 1981). O aumento da fosfatase alcalina no fluido peritoneal e a ausência de aumento correspondente no soro de equinos com distúrbios abdominais foram relatados por Frosher e Nagode (1979), o que assemelha-se aos achados deste ensaio.

Nesta pesquisa, também foi possível evidenciar que as alterações nas concentrações de fibrinogênio e da atividade da fosfatase alcalina são mensuradas precocemente, a partir das 6 horas p.o., no fluido peritoneal quando comparados com os valores obtidos no sangue circulante de animais submetidos a enterotomias no cólon descendente. Portanto, os períodos decisivos para a resolução do processo inflamatório abdominal em equinos submetidos a enterotomias do cólon descendente ocorre às 48 horas p.o. para a fosfatase alcalina e às 168 horas para o fibrinogênio do fluido peritoneal e, para o fibrinogênio plasmático às 336 horas p.o.

Comprova-se, assim, a importância das determinações seriadas do fibrinogênio plasmático, e do fluido peritoneal e da atividade da fosfatase alcalina no fluido peritoneal no período pós-operatório de equinos submetidos a enterotomias do cólon descendente. 
Tabela 2 - Valores referentes a média geral e desvio padrão para as atividades sérica e do fluido peritoneal da fosfatase alcalina (U/L) de equinos submetidos a enterorrafias invaginante (GI) e aposicional (GII) no cólon descendente, nos diferentes momentos de colheita.

(Continua)

\begin{tabular}{|c|c|c|c|c|c|c|}
\hline \multirow[b]{2}{*}{$\begin{array}{l}\text { TEMPO } \\
\text { (horas) }\end{array}$} & \multicolumn{3}{|c|}{ FOSFATASE ALCALINA SÉRICA (U/L) } & \multicolumn{3}{|c|}{$\begin{array}{l}\text { FOSFATASE ALCALINA DO FLUIDO } \\
\text { PERITONEAL (U/L) }\end{array}$} \\
\hline & $\begin{array}{c}\text { MGG } \\
\text { GRUPO I }\end{array}$ & $\begin{array}{c}\text { MGG } \\
\text { GRUPO II }\end{array}$ & MGT & $\begin{array}{c}\text { MGG } \\
\text { GRUPO I }\end{array}$ & $\begin{array}{c}\text { MGG } \\
\text { GRUPO II }\end{array}$ & MGT \\
\hline-24 & $\begin{array}{r}160,68 \\
\pm 64,29 \\
\end{array}$ & $\begin{array}{r}99,18 \\
\pm 50,07 \\
\end{array}$ & $\begin{array}{r}148,45 \\
\pm 73,25 \\
\end{array}$ & $\begin{array}{r}13,74 \\
\pm 8,27\end{array}$ & $\begin{array}{r}17,21 \\
\pm 15,36 \\
\end{array}$ & $\begin{array}{r}15,34 \\
\pm 11,65 \\
\end{array}$ \\
\hline 0 & $\begin{array}{r}189,83 \\
\pm 70,19 \\
\end{array}$ & $\begin{array}{c}101,43 \pm \\
53,05\end{array}$ & $\begin{array}{r}149,04 \\
\pm 75,76 \\
\end{array}$ & $\begin{array}{r}14,75 \\
\pm 12,00 \\
\end{array}$ & $\begin{array}{r}13,46 \\
\pm 12,85 \\
\end{array}$ & $\begin{array}{r}14,16 \\
\pm 11,88 \\
\end{array}$ \\
\hline 6 & $\begin{array}{l}196,98 \\
\pm 68,23 \\
\end{array}$ & $\begin{array}{l}161,20 \\
\pm 64,81 \\
\end{array}$ & $\begin{array}{l}180,47 \\
\pm 66,50 \\
\end{array}$ & $\begin{array}{l}266,77 \\
\pm 92,63 \\
\end{array}$ & $\begin{array}{r}468,35 \\
\pm 271,16 \\
\end{array}$ & $\begin{array}{r} \\
359,80 \\
\pm 214,48 \\
\end{array}$ \\
\hline 12 & $\begin{array}{r}172,08 \\
\pm 56,64 \\
\end{array}$ & $\begin{array}{r}162,46 \\
\pm 56,64 \\
\end{array}$ & $\begin{array}{r}167,64 \\
\pm 54,46 \\
\end{array}$ & $\begin{array}{r}612,18 \\
+465,91 \\
\end{array}$ & $\begin{array}{r}898,98 \\
\pm 500,02 \\
\end{array}$ & $\begin{array}{r}744,55 \\
\pm 484,62 \\
\end{array}$ \\
\hline 18 & $\begin{array}{r}172,57 \\
\pm 53,89 \\
\end{array}$ & $\begin{array}{r}157,10 \\
\pm 62,18 \\
\end{array}$ & $\begin{array}{r}165,43 \\
\pm 55,93 \\
\end{array}$ & $\begin{array}{r}729,78 \\
+1120,44 \\
\end{array}$ & $\begin{array}{r}857,76 \\
\pm 553,95 \\
\end{array}$ & $\begin{array}{r}788,85 \\
\pm 871,76 \\
\end{array}$ \\
\hline 24 & $\begin{array}{r}165,14 \\
\pm 37,50 \\
\end{array}$ & $\begin{array}{r}143,13 \\
\pm 65,48 \\
\end{array}$ & $\begin{array}{r}154,98 \\
\pm 51,19 \\
\end{array}$ & $\begin{array}{r}411,25 \\
+296,67 \\
\end{array}$ & $\begin{array}{r}861,00 \\
\pm 774,38 \\
\end{array}$ & $\begin{array}{r}619,20 \\
\pm 590,10 \\
\end{array}$ \\
\hline 48 & $\begin{array}{r}148,73 \\
\pm 37,46 \\
\end{array}$ & $\begin{array}{r}113,56 \\
\pm 40,03 \\
\end{array}$ & $\begin{array}{r}132,50 \\
\pm 41,26 \\
\end{array}$ & $\begin{array}{c}182,23 \\
\pm 170,13\end{array}$ & $\begin{array}{r}320,70 \\
\pm 358,21\end{array}$ & $\begin{array}{c}246,13 \\
\pm 270,37\end{array}$ \\
\hline 72 & $\begin{array}{r}146,67 \\
\pm 41,37 \\
\end{array}$ & $\begin{array}{r}118,63 \\
\pm 47,08 \\
\end{array}$ & $\begin{array}{r}133,73 \\
\pm 44,62 \\
\end{array}$ & $\begin{array}{c}209,64 \\
\pm 260,50 \\
\end{array}$ & $\begin{array}{r}252,20 \\
\pm 193,20 \\
\end{array}$ & $\begin{array}{c}229,28 \\
\pm 223,49 \\
\end{array}$ \\
\hline 96 & $\begin{array}{r}143,68 \\
\pm 36,11 \\
\end{array}$ & $\begin{array}{r}110,05 \\
\pm 25,67 \\
\end{array}$ & $\begin{array}{r}128,13 \\
\pm 35,07 \\
\end{array}$ & $\begin{array}{c}176,21 \\
\pm 239,26 \\
\end{array}$ & $\begin{array}{r}180,55 \\
\pm 112,83 \\
\end{array}$ & $\begin{array}{c}178,21 \\
\pm 184,21 \\
\end{array}$ \\
\hline 168 & $\begin{array}{r}143,31 \\
\pm 35,57 \\
\end{array}$ & $\begin{array}{r}118,20 \\
\pm 38,75 \\
\end{array}$ & $\begin{array}{r}131,72 \\
\pm 37,79 \\
\end{array}$ & $\begin{array}{r}100,51 \\
\pm 125,43 \\
\end{array}$ & $\begin{array}{r}64,90 \\
\pm 61,29 \\
\end{array}$ & $\begin{array}{c}84,07 \\
\pm 98,86 \\
\end{array}$ \\
\hline 336 & $\begin{array}{l}171,08 \\
\pm 61,22\end{array}$ & $\begin{array}{r}116,60 \\
\pm 31,00\end{array}$ & $\begin{array}{r}145,94 \\
\pm 55,04\end{array}$ & $\begin{array}{c}53,14 \\
\pm 32,90\end{array}$ & $\begin{array}{c}25,41 \\
\pm 16,31\end{array}$ & $\begin{array}{r}40,34 \\
\pm 29,31\end{array}$ \\
\hline 504 & $\begin{array}{r}151,98 \\
\pm 61,57 \\
\end{array}$ & $\begin{array}{r}98,60 \\
\pm 40,29 \\
\end{array}$ & $\begin{array}{r}127,34 \\
\pm 57,78 \\
\end{array}$ & $\begin{array}{c}30,91 \\
\pm 22,43 \\
\end{array}$ & $\begin{array}{r}74,86 \\
\pm 160,16 \\
\end{array}$ & $\begin{array}{r}51,20 \\
+107,05 \\
\end{array}$ \\
\hline 672 & $\begin{array}{r}123,31 \\
\pm 37,67\end{array}$ & $\begin{array}{r}107,25 \\
\pm 29,02\end{array}$ & $\begin{array}{r}115,90 \\
\pm 33,62\end{array}$ & $\begin{array}{l}20,21 \\
\pm 9,88\end{array}$ & $\begin{array}{r}43,73 \\
\pm 82,02\end{array}$ & $\begin{array}{c}31,07 \\
\pm 54,78\end{array}$ \\
\hline 840 & $\begin{array}{r}123,90 \\
\pm 27,07 \\
\end{array}$ & $\begin{array}{r}140,08 \\
\pm 67,39\end{array}$ & $\begin{array}{l}131,37 \\
\pm 48,26 \\
\end{array}$ & $\begin{array}{c}23,07 \\
\pm 12,62 \\
\end{array}$ & $\begin{array}{c}23,03 \\
+37,05 \\
\end{array}$ & $\begin{array}{c}23,05 \\
\pm 25,53 \\
\end{array}$ \\
\hline
\end{tabular}


Tabela 2 - Valores referentes a média geral e desvio padrão para as atividades sérica e do fluido peritoneal da fosfatase alcalina (U/L) de equinos submetidos a enterorrafias invaginante (GI) e aposicional (GII) no cólon descendente, nos diferentes momentos de colheita.

(Conclusão)

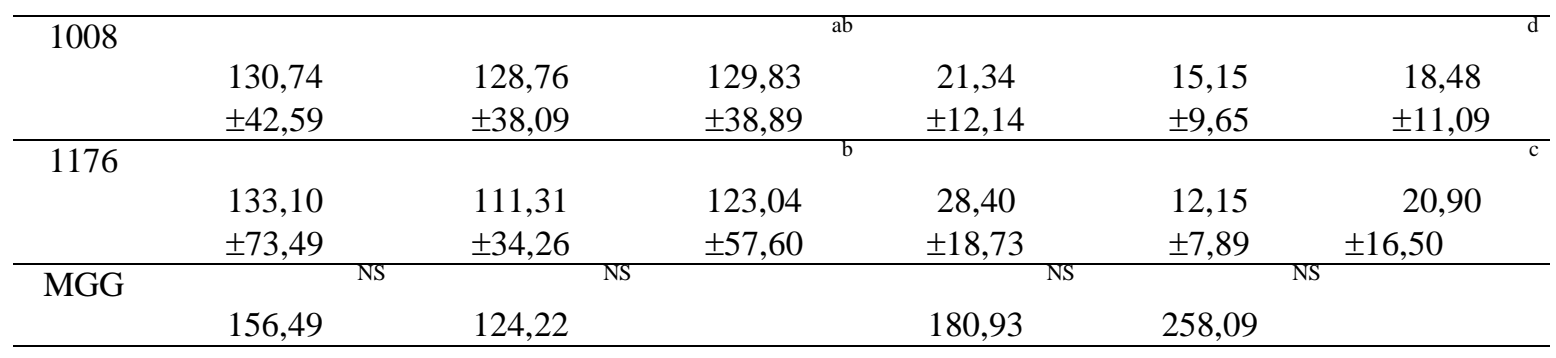

Médias seguidas pela mesma letra não diferem entre si (Teste Tukey, $\mathrm{p} \square 0,05$ )

Letras minúsculas diferentes representam diferença $(\mathrm{P} \leq 0,05)$ entre momentos

MGT= média geral dos grupos em cada momento

MGG= média geral de cada grupo em todos os momentos

\section{CONCLUSÃO}

As avaliações seriadas das concentrações de fibrinogênio plasmático e do fibrinogênio e da fosfatase alcalina do fluido peritoneal foram de grande valor no entendimento da dinâmica inflamatória, caracterizando a existência de um processo inflamatório agudo intra-abdominal nos animais submetidos às técnicas de enterorrafias invaginante e aposicional.

A inflamação da cavidade abdominal foi evidenciada precocemente (6 horas p.o.) com as análises do fibrinogênio e da fosfatase alcalina do fluido peritoneal e, mais tardiamente, pelo fibrinogênio plasmático (18 horas p.o.), sendo úteis no acompanhamento da resolução do processo inflamatório intra-abdominal e no prognóstico de equinos submetidos a enterotomias no cólon descendente.

\section{AGRADECIMENTOS}

À Fundação de Amparo à Pesquisa do Estado de São Paulo (FAPESP) pelo apoio financeiro.

Aos Técnicos de Laboratório Eugênio de Campos Filho, Cláudia Aparecida da Silva Nogueira e Renata Lemos Nagib Jorge, do Departamento de Clínica e Cirurgia Veterinária da FCAV, UNESP, Jaboticabal, pelo auxílio nas análises bioquímicas. 
Ao funcionário Carlos Roberto Januário do setor de grandes animais do Departamento de Clínica e Cirurgia Veterinária Hospital Veterinário “Governador Laudo Natel” da FCAV, UNESP, Jaboticabal, pelo auxílio técnico.

\section{REFERÊNCIAS}

ARCHER, R.M. et al. A comparison of enterotomies though the antimesenteric band and the sacculation of small (descending) colon of ponies. Equine Vet. J., v.20, n.6, p.406-413, 1988.

BACCARIN, R.Y.A. et al. Alterações do líquido peritoneal em eqüinos com desconforto abdominal e suas relações com o tipo de lesão implantada e evolução após tratamento médico ou cirúrgico: análise de 70 casos. Braz. J. Vet. Res. Anim. Sci., v.32, n.4, p.256-265, 1995.

BARRELET, A. Peritoneal fluid: Part I- Laboratory analyses. Equine Vet. Educ., v.5, n.2, p.81-83, 1983.

BLACKFORD, J.T. et al. Equine peritoneal fluid analysis following celiotomy. In: EQUINE COLIC RESEARCH SYMPOSIUM, 2., Lawrenceville, 1986. Proceedings... Lawrenceville, 1986. p.130-132.

BROWNLOW, M.A.; HUTCHINS, D.R.; JOHNSTON, K. G. Reference values for equine peritoneal fluid. Equine Vet. J., v.13, n.2, p.127-130, 1981.

COFFMAN, J., The plasma proteins. Vet. Med., p. 1168-1170, 1979.

DAVIES, J.V. et al. Experimental ischaemia of the ileum and concentrations of thee intestinal isoenzyme of alkaline phosphatase in plasma and peritoneal fluid. Equine Vet. J., v.16, n.3, p.215-217, 1984.

EDWARDS, G.B. Diseases and surgery of the small colon. Vet. Clin of North Am., v.13, n.2, p.359-375, 1997.

FALEIROS, R. Diagnóstico precoce da desvitalização de segmento do cólon menor equiino submetido experimentalmente à distensão intraluminal, 2003. p. 29-57. Tese (Doutorado em Cirurgia Veterinária) - Faculdade de Ciências Agrárias e Veterinárias, Universidade Estadual Paulista, Jaboticabal.

FISHER JR., A.T et al. Diagnostic laparoscopy in the horse. J. Am. Vet. Med. Assoc., v.189, n.3, p.289-292, 1986.

FROSCHER B.G.; NAGODE, L.A. Isoenzymes of equine alkaline phosphatase. Am. J. Vet. Res., v.40, n.11, p.1514-1517, 1979.

GODBER, L.M. et al. Pharmacocinetics, nephrotoxicoses and in vitro antibacterial activity associated with single versus multiple (three times) daily gentamicin treatments in horses. Am. J. Vet. Res., v.56, n.5, p.613-618, 1995. 
HAWKINS, J.F. et al. Peritonitis in horses: 67 cases (1985-1990). J. Am. Vet. Med. Assoc., v.203, n.2, p.284-287, 1993.

JAIN, N.C. Schalm's veterinary hematology. Philadelphia: Lea \& febiger, 1986. $1221 \mathrm{p}$.

KANEKO, J. Clinical biochemistry of domestic animals. San Diego: Academic Press, 1989. 932p.

LUCAS, F.A et al. Estudo comparativo da cicatrização de enterorrafias em planos aposicional e invaginante no cólon descendente de eqüinos, Arq. Bras. Med. Vet. Zootec., v.53, n.5, p.577-584, 2001.

MALARK, J.A.; PEYTON, L.C.; GALVIN, M.J. Effects of blood contamination on eqüine peritoneal fluid analysis. J. Am. Vet. Med. Assoc., v.201, n.10, p.1545-1556, 1992.

McILWRAITH, C.W.; TURNER, A.S. Use of prophylactic antibiotis in equine surgery. In: Equine surgery advanced techniques. Philadelphia: Lea \& Febiger, 1987. p.12-19.

PARRY, B.W.; BROWNLOW, M.A. Peritoneal Fluid. In: COWELL R.L.; TYLER, R.D. Citology and hematology of the horse. California: American Veterinary, 1992. p.121-151.

PEIRÓ, J.R. Endotoxemia experimental em equinos: avaliação clínico-laboratorial. Jaboticabal. 1997. 89p. Dissertação (Mestrado em Cirurgia Veterinária) Faculdade de Ciências Agrárias e Veterinárias, Universidade Estadual Paulista, Jaboticabal.

SANTSCHI, E.M. et al. Peritoneal fluid analysis in ponies after abdominal surgery. Vet. Surg., v.17, n.1, p.6-9, 1988.

SCHNEIDER,R. K. et al. Response of peritoneum to four lavage peritoneal solutions, Am. J. Vet. Res. v.49, n.6, p.889-894, 1988.

SILVA, C.F.G.K.T. Valores hematológicos, bioquímicos e exame do líquido peritoneal de equiinos (Equus caballus, Linnaeus, 1758) durante síndrome cólica, 2005. 81p. Dissertação (Mestrado em Clínica Veterinária) Fac. de Medicina Veterinária e Zootecnia, Universidade Estadual Paulista, Botucatu.

STASHAK,T.S. Techniques for enterotomy, descompression, and intestinal resection/ anastomosis. Vet. Clin. North Am.: Large Anim. Pract., v.4, n.1, p.148-165, 1982.

TULLENERS, E.P. Complications of abdominocentesis in the horse. J. Am. Vet. Med. Assoc., v.182, n.3, p.232-234, 1983.

TURNER, A.S.; McILWRAITH, C.W. Equine dental and gastrointestinal surgery. Techniques in large animal surgery. Philadelphia: Lea \& Febiger, 1982. p.211-216.

VALADÃO, C.A.; ÁVILA JÚNIOR, O.S.; CAMPOS FILHO, E. Aspectos bioquímicos do plasma e fluido peritoneal de eqüinos com cólica. Braz. J. Vet. Res. anim. Sci., v.33, p.32-35, 1996.

WHITE II, N.A. The equine acute abdomen. Philadelphia: Lea \& febiger, 1990. 443p. 